\title{
Glueballs and deconfinement temperature in AdS/QCD
}

\author{
S. S. Afonin ${ }^{*}$ and A. D. Katanaeva ${ }^{\dagger}$ \\ Saint Petersburg State University, 7/9 Universitetskaya naberezhnaya, St. Petersburg, 199034, Russia
}

(Received 24 September 2018; published 21 December 2018)

\begin{abstract}
We put forward an alternative way to estimate the deconfinement temperature in bottom-up holographic models. The deconfinement in anti-de Sitter (AdS)/QCD is related to the Hawking-Page phase transition, in which the critical Hawking temperature is identified with the deconfinement one in the gauge theory. The numeric estimation of the latter is only possible when the parameters of a five-dimensional dual model have been previously determined from consistency with other physical aspects, standardly, providing a description of the QCD resonances. The traditional way to fix parameters in the simplest AdS/QCD models is to reproduce the mass of the $\rho$ meson or the slope of the approximate radial Regge trajectory of the $\rho$ excitations. Motivated by a general idea that the slope value originates in gluodynamics, we propose calculating the deconfinement temperature using the trajectory of scalar glueballs. We consider several holographic models and use the recent idea of isospectral potentials to make an additional check of the relevance of our approach. It is demonstrated that different models from an isospectral family (i.e., the models leading to identical predictions for the spectrum of hadrons with fixed quantum numbers) result in different predictions for the deconfinement temperature. This difference is found to be quite small in the scalar glueball channel but very large in the vector meson channel. The observed stability in the former case clearly favors the choice of the glueball channel for thermodynamic predictions in AdS/QCD models. For a balanced approach, we argue that either assuming $f_{0}(1500)$ to have a dominating component of $0^{++}$ glueball or accepting the idea of the universality in the radial Regge trajectories of light nonstrange vector mesons one can reproduce the results for the deconfinement temperature obtained before in the lattice simulations in the background of nondynamical quarks.
\end{abstract}

DOI: 10.1103/PhysRevD.98.114027

\section{INTRODUCTION}

The anti-de Sitter (AdS)/QCD correspondence proved to be a useful technique to study different facets of the strongly coupled theories. Besides the established QCD content of mesons and baryons, one may try to apply the holography to the hypothesized QCD bound states-the glueballs.

Introduced in the 1970s [1,2], these particles continue to be a subject of intense study both in lattice QCD and in various phenomenological models. Experimental searches are carried out (BES III and Belle II), but no unambiguous identification may be claimed (see, for instance, the review "non- $q \bar{q}$ Mesons" in Particle Data [3] or Ref. [4]). Future experiments, such as PANDA at the FAIR facility and

\footnotetext{
s.afonin@spbu.ru

alice.katanaeva@gmail.com
}

Published by the American Physical Society under the terms of the Creative Commons Attribution 4.0 International license. Further distribution of this work must maintain attribution to the author(s) and the published article's title, journal citation, and DOI. Funded by SCOAP ${ }^{3}$. several programs at NICA, are expected to specify the existing hypotheses (see, e.g., suggestions in Refs. [5,6]).

Indeed, the holographic investigation of glueballs has been performed before: from first applications of the Maldacena conjecture (e.g., in Ref. [7]) and top-down realizations (e.g., in Refs. [8,9]), to bottom-up phenomenological approaches implemented in Refs. [10-13] and more recent ones, like Ref. [14], introducing more complicated models. The focus of these papers mostly stays on predictions of the spectrum and decay rates, as well as on the corroboration of the holographic correlation functions in the light of other existing QCD techniques. In this work, we would like to sidestep and provide the connection of this kind of bottom-up models to another pressing issue of holographic QCD.

The QCD phase diagram, another issue of interest in strong interactions, is subject to holographic treatment as well. Within the bottom-up approach, this type of study was pioneered by C. P. Herzog in Ref. [15] and continued by many authors (see, e.g., Ref. [16] and references therein). In these papers, the five-dimensional (5D) model is constructed from the point of view that the gravity part of the 5D action describes the gluodynamic background and thermodynamic properties of the 4D holographic dual. 
The Hawking-Page-type phase transition between different AdS backgrounds may happen at some critical temperature $T_{c}$, and then this characteristic is interpreted as the temperature of transition from the confined to the deconfined phase. Additionally, the 5D matter content represents the 4D states bound by the strong interactions.

The two sectors of the 5D model turn out to be closely intertwined when concerning the value of the deconfinement temperature. The estimation of the last depends on the model parameters that "historically" were connected to the dominant purpose of the first bottom-up AdS/QCD papers (such as the ones of Refs. [17-19]) - to the description of light vector meson spectra. The estimates of $T_{c}$ in Ref. [15] follow the parameter values of these works. Though this is a traditional choice, there seems to be no particular reason why the vector meson spectra should in any way determine the deconfinement temperature. At the same time, particle spectra, especially the radial Regge trajectories appearing in soft wall (SW) models, are one of the most attractive features of holography.

We would like to propose a different kind of particle-a spin-0 glueball (and its radial excitations) - to fix the matter part of the holographic model and define the model parameters. To vindicate the concept, we provide several "pro" arguments in the text, which may be shortly given as follows:

(i) The phase diagram can be studied in pure gluodynamics. Since the holographic approach is defined in the large- $N_{c}$ (planar) limit of gauge theories in which the glueballs dominate over the usual mesons and baryons (as the quarks are in the fundamental representation), the gluodynamics must dictate the overall mass scale and thereby the major contribution to the deconfinement temperature $T_{c}$.

(ii) Within the isospectrality concept [20], one can show that the predicted values of $T_{c}$ are more stable for scalar glueballs than for vector mesons.

(iii) Considering phenomenological reasons, numerical values of $T_{c}$ determined in the scalar glueball framework can be interpreted as better fitting the lattice expectations.

The first argument was scrutinized in our paper [16], and we will further substantiate the point. One can observe, e.g., that if we take the linear radial spectrum of scalar glueballs given by the standard SW holographic model, $m_{n}^{2}=\mu^{2}(n+2), n=0,1,2, \ldots$ (the interpolating operator $G_{\mu \nu}^{2}$ is assumed [11]), and consider the scalar resonance $f_{0}(1500)$ [3] as the lightest glueball (as is often proposed in the hadron spectroscopy [3]), we will obtain the slope $\mu^{2}=1.13 \mathrm{GeV}^{2}$. It agrees perfectly with the mean radial slope $\mu^{2}=1.14 \pm 0.01 \mathrm{GeV}^{2}$ found for the light mesons in the analysis [21] and achieved independently in Refs. [22,23]. In Ref. [16], we showed that this value of $\mu^{2}$ leads to a consistent numerical estimation of the deconfinement temperature in the SW model.
The second argument is motivated by an interesting finding of the authors of Ref. [20] that the particular form of spectrum in SW-like models does not fix a model itself; one can always construct a one-parametric family of SW models (controlling modifications of the "wall") leading to the same spectrum. We will demonstrate that such isospectral models, however, result in different predictions for the deconfinement temperature $T_{c}$. In the vector channel originally considered by Herzog in Ref. [15], these variations can be quite significant, whereas in the scalar case, the difference may be rather small and span an interval admissible by the accuracy of the large $N_{c}$ limit.

The third argument is just a phenomenological observation for typical predictions of $T_{c}$ in the bottom-up holographic models. For instance, the original Herzog analysis of the vector hard wall (HW) holographic model with the $\rho$-meson taken as the lowest state resulted in the prediction $T_{c}=122 \mathrm{MeV}$ [15]. If we apply this analysis to the scalar HW model with $f_{0}(1500)$ as the lightest glueball, we will find $T_{c} \approx 150 \mathrm{MeV}$. The lattice simulations typically predict the lightest glueball near $1.7 \mathrm{GeV}$ (1.6-1.7 GeV as quoted in Particle Data Group reviews [3]). This value shifts the prediction to $T_{c} \approx 170 \mathrm{MeV}$. So, we may regard the interval $T_{c}=150-170 \mathrm{MeV}$ as a prediction of the HW model in the glueball channel. We find remarkable that exactly this interval was found in the modern lattice simulations with dynamical quarks [24].

In the present paper, we will consider scalar glueballs and vector mesons in parallel in order to demonstrate in detail the emergent differences. In Sec. II, we recall the general procedure to achieve an estimation of the deconfinement temperature in AdS/QCD models. Then, we provide some particular calculations in various not overcomplicated 5D frameworks in Sec. III. We introduce the notion of isospectrality in Sec. IV and show how it can affect the temperature values. In Sec. V, we briefly review lattice and experimental results in the relevant topics, mostly to provide benchmarks and input parameters for the models considered. Finally, in Sec. VI, we give our numerical estimations of $T_{c}$ distinguishing the glueball and vector meson channels and several different options within. We conclude in Sec. VII with a discussion on the general validity of the presented approach.

\section{HAWKING TEMPERATURE IN BOTTOM-UP MODELS}

Consider a general 5D action that contains a universal gravitational part and a matter part to be specified further and has an AdS related metric $g_{M N}\left(g=\operatorname{det} g_{M N}\right)$ :

$$
\begin{gathered}
S=\int d^{4} x d z \sqrt{-g} f^{2}(z)\left(\mathcal{L}_{\text {gravity }}+\mathcal{L}_{\text {matter }}\right) \\
\mathcal{L}_{\text {gravity }}=-\frac{1}{2 k_{g}}(\mathcal{R}-2 \Lambda) .
\end{gathered}
$$


Here, $k_{g}$ is the coefficient proportional to the 5D Newton constant, $\mathcal{R}$ is the Ricci scalar, and $\Lambda$ is the cosmological constant. The choice of the dilaton background, $f(z)$, distinguishes possible holographic models. They differ as well by the interval the $z$ coordinate spans. For now, we assume $z \in\left[0, z_{\max }\right]$, though $z_{\max }=\infty$ is possible and will be of the main interest in the present work.

The assessment of the critical temperature is related to the leading contribution in the large- $N_{c}$ counting, that is, the $\mathcal{L}_{\text {gravity }}$ part scaling as $\frac{1}{2 k_{g}} \sim N_{c}^{2}$. $\mathcal{L}_{\text {matter }}$ scales as $N_{c}$ and thus does not explicitly affect the confinement/deconfinement process.

It is found that the deconfinement in AdS/QCD occurs as a Hawking-Page phase transition [15]. Let us recall how the order parameter of this transition is defined for a given theory.

First, we should evaluate the free action densities $V$, being the regularized $S_{\text {grav }}$, on different backgrounds corresponding to two phases. One assumes that the thermal AdS of radius $R$ is defined by the general AdS line element

$$
d s^{2}=\frac{R^{2}}{z^{2}}\left(d t^{2}-d \vec{x}^{2}-d z^{2}\right),
$$

with the time direction restrained to the interval $[0, \beta]$. This background corresponds to the confined phase. The metric of the Schwarzschild black hole in AdS describes the deconfined phase and is given by

$$
d s^{2}=\frac{R^{2}}{z^{2}}\left(h(z) d t^{2}-d \vec{x}^{2}-\frac{d z^{2}}{h(z)}\right),
$$

where $h(z)=1-\left(z / z_{h}\right)^{4}$ and $z_{h}$ denotes the horizon of the black hole.

With the cosmological constant in 5D AdS being $\Lambda=-6 / R^{2}$, both these metrics are the solutions of the Einstein equations and provide the same value of the Ricci scalar $\mathcal{R}=-20 / R^{2}$. Hence, the free action densities differ only in the integration limits,

$$
\begin{gathered}
V_{\mathrm{Th}}(\epsilon)=\frac{4 R^{3}}{k_{g}} \int_{0}^{\beta} d t \int_{\epsilon}^{z_{\max }} d z f^{2}(z) z^{-5}, \\
V_{\mathrm{BH}}(\epsilon)=\frac{4 R^{3}}{k_{g}} \int_{0}^{\pi z_{h}} d t \int_{\epsilon}^{\min \left(z_{\max }, z_{h}\right)} d z f^{2}(z) z^{-5} .
\end{gathered}
$$

The two geometries are compared at $z=\epsilon$, where the periodicity in the time direction is locally the same, i.e., $\beta=\pi z_{h} \sqrt{h(\epsilon)}$. Then, we may construct the order parameter for the phase transition,

$$
\Delta V=\lim _{\epsilon \rightarrow 0}\left(V_{\mathrm{BH}}(\epsilon)-V_{\mathrm{Th}}(\epsilon)\right) .
$$

The thermal AdS is stable when $\Delta V>0$; otherwise, the black hole is stable. The condition $\Delta V=0$ defines the critical temperature $T_{c}$ at which the transition between the two phases happens through the definition of the Hawking temperature $T=1 /\left(\pi z_{h}\right)$.

However, to provide a numerical estimation of $T_{c}$, the usage of Eq. (7) is not enough as it yields $z_{h}$ as a function of model-dependent parameters $-z_{\max }$ and/or those possibly introduced in $f(z)$. We must appeal to the matter sector $\mathcal{L}_{\text {matter }}$ to give physical meaning to these parameters and to connect $T_{c}$ to a particular type of a holographic model.

\section{INTRODUCING MATTER CONTENT IN VARIOUS 5D MODELS}

Following the stated route, we would like to compare the results for the deconfinement temperature in different cases, whether we consider a matter content of vector mesons or scalar glueballs. We provide them in parallel, browsing through the general features first.

The $\mathcal{L}_{\text {matter }}$ for an Abelian (for simplicity) vector field $A_{M}(x, z)$ and a scalar field $\varphi(x, z)$ are given by

$$
\begin{gathered}
\mathcal{L}_{v}=-\frac{1}{4 g_{5}^{2}} g^{M P} g^{N Q}\left(\partial_{M} A_{N}-\partial_{N} A_{M}\right)\left(\partial_{P} A_{Q}-\partial_{Q} A_{P}\right), \\
\mathcal{L}_{s c}=\frac{1}{2 k_{s}}\left(g^{M N} \partial_{M} \varphi \partial_{N} \varphi-M_{5}^{2} \varphi^{2}\right),
\end{gathered}
$$

where $k_{s}$ and $g_{5}$ are the normalization constants. The 5D field $\varphi$ is dual to $G_{\mu \nu} G^{\mu \nu}$ - the lowest-dimension QCD operator that bears the scalar glueball quantum numbers $0^{++}$. The general methods of the AdS/CFT conjecture prescribe $M_{5}^{2} R^{2}=0$ as the conformal dimension of the dual 4D operator is 4 . The vector field $A_{M}$ is dual to the QCD conserved current $\bar{q} \gamma_{\mu} q$ of dimension 3. AdS/CFT prescribes such a vector $5 \mathrm{D}$ field to be massless as well. The standard holographic gauge choice is $A_{z}=0$.

In the following, if possible, we are going to use the unified notation for both cases introducing the spin parameter $J$, with $J=0$ corresponding to the scalar case and $J=1$ corresponding to the vector one. ${ }^{1}$

Let us call the general spin 5D field $\Phi(x, z)$ (we suppress the Lorentz indices). Then, the equations of motion (EOM) for the Fourier transformation of these arbitrary spin 5D fields read as follows [25]:

$$
\begin{gathered}
\partial_{z} \frac{f^{2}(z)}{z^{3-2 J}} \partial_{z} \Phi(q, z)-\frac{M_{5}^{2} R^{2}}{z^{5-2 J}} f^{2}(z) \Phi(q, z) \\
+\frac{f^{2}(z)}{z^{3-2 J}} q^{2} \Phi(q, z)=0
\end{gathered}
$$

\footnotetext{
${ }^{1}$ We presume the method to be valid for any spin, under condition that the 5D action is of the form discussed in Ref. [25]. Nevertheless, there exists no clear notion on the treatment of higher-spin states in the literature. See the different approach in Ref. [19], though it fails to provide the correct result for scalar fields and hence cannot be used by us.
} 
Further, we consider $M_{5}^{2} R^{2}=0$ as befits our models. In Ref. [25], it is discussed that for higher-spin fields the term coming with $M_{5}^{2}$ may turn out to be $z$ dependent and it is a condition of light front holographic QCD that it takes a particular constant value. We will not go into this discussion, as for our cases of vector and scalar Lagrangians, Eq. (10) provides the conventional EOM with $M_{5}^{2} R^{2}$ defined from the holographic dictionary as mentioned above.

The standard procedure to find the spectrum is to solve EOM as the eigenvalue problem imposing $q^{2}$ to take the discrete values $q^{2}=M^{2}(n)(n=0,1,2 \ldots$ being a discrete parameter). Then, the Kaluza-Klein (KK) decomposition is given as

$$
\Phi(q, z)=\sum_{n=0}^{\infty} \phi_{n}(z) \phi^{(n)}(q) .
$$

$\phi^{(n)}$ represents the tower of 4D excitations. Their $z$ profiles are determined by demanding specific boundary conditions in the $z$ direction. Thus, further investigation requires a specification of the model.

\section{A. HW option}

Let us start with the simplest option-the hard wall model $[17,18]$. This model is characterized by $f^{2}(z)=1$ and an explicit cutoff of the $z$ direction at some finite position $z_{\max }$. The $z$-dependent solution of Eq. (10) $\phi_{n}(z)$ should be subject to the Dirichlet boundary condition in the UV $\phi_{n}(0)=0$ and the Neumann one on the IR cutoff $\partial_{z} \phi_{n}\left(z_{\max }\right)=0$. The appropriate solution is

$$
\Phi(q, z) \sim(q z)^{2-J} J_{|2-J|}(q z),
$$

where $J_{\alpha}$ is a Bessel function of the first type. As the recurrence relation for Bessel functions states $\partial_{z}\left(z^{\alpha} J_{\alpha}\right)=$ $z^{\alpha} J_{\alpha-1}$, the IR boundary condition translates into $J_{\alpha-1}\left(M(n) z_{\max }\right)=0$. The first zeros of $J_{0}$ and $J_{1}$ fix the values of the ground states as

$$
\begin{aligned}
& m_{\mathrm{HW}}^{J=1}=M_{J=1}(0)=\frac{2.405}{z_{\max }}, \\
& m_{\mathrm{HW}}^{J=0}=M_{J=0}(0)=\frac{3.832}{z_{\max }} .
\end{aligned}
$$

Concerning the particular consequences for the order parameter of the Hawking-Page phase transition, the result of Eq. (7) in the HW model is

$$
\Delta V_{\mathrm{HW}}=0 \Leftrightarrow z_{\max }^{4}=2 z_{h}^{4},
$$

as has been found in Ref. [15]. Then, the deconfinement temperature is determined as

$$
\begin{aligned}
& T_{\mathrm{HW}}^{J=1}=\frac{2^{1 / 4}}{\pi z_{\max }}=\frac{2^{1 / 4} m_{\mathrm{HW}}^{J=1}}{2.405 \pi}=0.1574 m_{\mathrm{HW}}^{J=1}, \\
& T_{\mathrm{HW}}^{J=0}=\frac{2^{1 / 4}}{\pi z_{\max }}=\frac{2^{1 / 4} m_{\mathrm{HW}}^{J=0}}{3.832 \pi}=0.0988 m_{\mathrm{HW}}^{J=0} .
\end{aligned}
$$

Evidently, these values have different numerical prefactors and depend on the mass of the first resonances, which do not have the same physical origin. Hence, we cannot expect to get a universal estimation of $T_{c}$ in HW models; for the numerics, see Sec. VI.

\section{B. (G)SW option}

The main achievement of the soft wall model [19] was the reproduction of the linear Regge and radial trajectories for mesons. It is natural to hypothesize that a scalar glueball and its radial excitation may lie on the linear trajectory as well.

The traditional SW model is characterized by an infinite IR cutoff $z_{\max }=\infty$, and the conformality is broken by the introduction of the dilaton profile $f^{2}(z)=e^{-\kappa^{2} z^{2}}$. On the UV brane, the Dirichlet condition is imposed, and good convergence is required in the IR (to be suppressed by the dilaton exponent). The normalizable discrete modes of Eq. (10) are

$$
\phi_{n}(z)=\mathcal{N}_{n}(\kappa z)^{2-J+|2-J|} L_{n}^{|2-J|}\left(\kappa^{2} z^{2}\right),
$$

where $L_{n}^{m}$ are the generalized Laguerre polynomials and $\mathcal{N}_{n}$ are the normalization factors of no importance to us. For the discrete parameter $n=0,1,2, \ldots$, we obtain the SW spectra,

$$
M_{J}^{2}(n)=4 \kappa^{2}\left(n+1+\frac{|2-J|-J}{2}\right)
$$

where one should choose $J=0$ or 1 , and $M_{5}^{2} R^{2}=0$ is assumed from the beginning.

A natural generalization toward a vector meson or glueball spectrum with an arbitrary intercept parameter $b$,

$$
M_{J}^{2}(n)=4 \kappa^{2}\left(n+1+b+\frac{|2-J|-J}{2}\right),
$$

may be achieved using the generalized soft wall (GSW) profile proposed in Ref. [26] (see also Ref. [27]):

$$
f^{2}(z)=e^{-\kappa^{2} z^{2}} U^{2}\left(b, J-1 ; \kappa^{2} z^{2}\right) .
$$

The modification consists in the Tricomi hypergeometric function $U$ that provides the necessary free parameter $b$ to the spectrum but does not change the SW asymptotes in UV and IR. As $U(0, J-1 ; x)=1$, the GSW with $b=0$ reduces to the usual SW. 

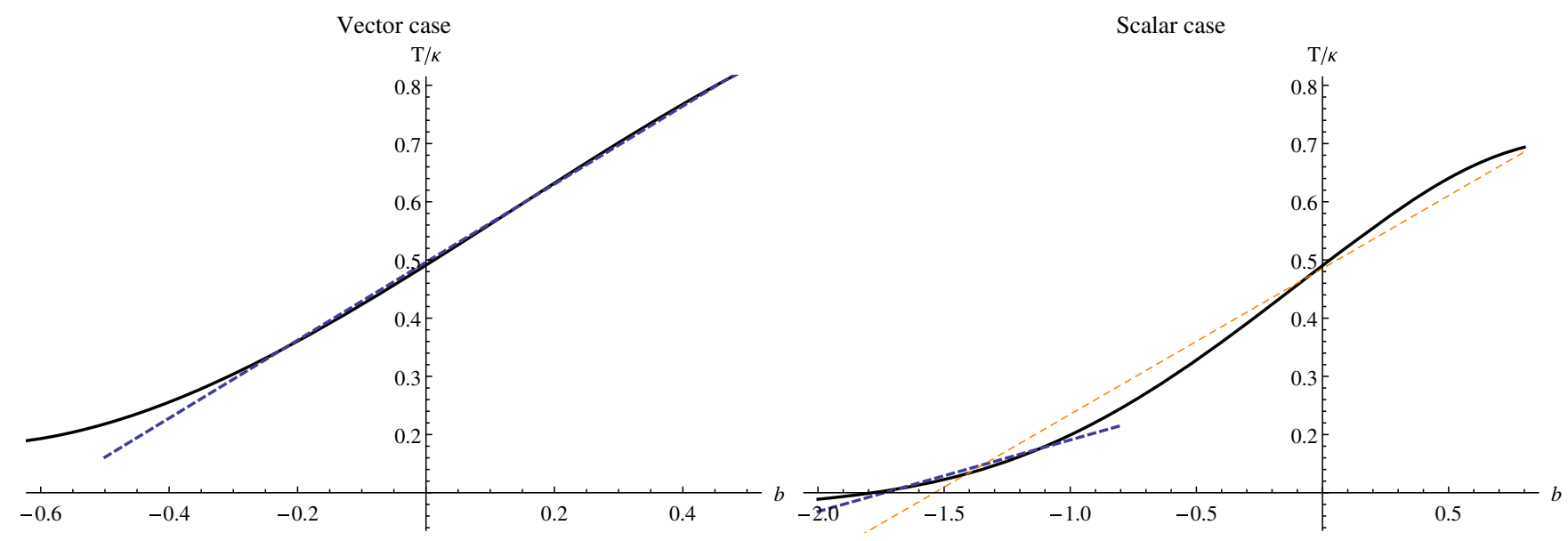

FIG. 1. The deconfinement temperature in the GSW model as a function of the intercept parameter $b$. The blue dashed lines are the linear interpolation functions given in Eq. (25).

The estimation of $\Delta V$ is similar to the one performed in Ref. [16] (though there the $U$ function has a fixed second parameter, adjusting to the vector spectrum) and results in

$$
\begin{aligned}
\Delta V_{\mathrm{GSW}}= & \frac{\pi R^{3}}{2 k_{g} z_{h}^{3}}\left[U^{2}(b, J-1 ; 0)-4\left(\kappa z_{h}\right)^{4}\right. \\
& \left.\times \int_{\kappa^{2} z_{h}^{2}}^{\infty} d t e^{-t} t^{-3} U^{2}(b, J-1 ; t)\right] .
\end{aligned}
$$

Taking $b=0$, one easily recovers the SW model result

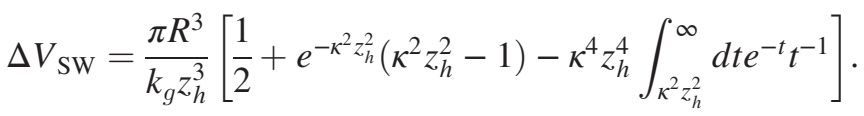

Numerically, one can find the value of $z_{h}$, as a function of $\kappa$ (and $b$ ), that solves the equation $\Delta V_{(G) S W}=0$. For the simple SW, we may reproduce the expression of the deconfinement temperature from [15]

$$
T_{\mathrm{SW}} \simeq 0.49 \cdot \kappa .
$$

In the GSW case, the following numerical approximations are valid for the values of $b$ corresponding to phenomenological spectra (to be discussed in Sec. V) (see Fig. 1):

$$
\begin{aligned}
& T_{\mathrm{GSW}}^{J=1} / \kappa \simeq 0.670 \cdot b+0.496, \\
& T_{\mathrm{GSW}}^{J=0} / \kappa \simeq 0.123 \cdot b+0.314 .
\end{aligned}
$$

Again, we postpone the discussion of numerics to Sec. VI.

An interesting but speculative feature of the SW model is the usage of the inverse dilaton profile [28], which means the case $f^{2}(z)=e^{\kappa^{2} z^{2}}$. Among other things, this choice modifies the intercept of the particle spectra. In the context of the AdS/QCD study of glueballs, the inverse dilaton option is sometimes considered. For instance, in Ref. [14], a modified SW model is investigated, and the negative dilaton supposedly provides the best fit for the scalar glueball masses. From the point of view of thermodynamic properties, we have to argue that the inverse dilaton puts the theory in the permanently deconfined (black hole) phase. It is straightforward to reproduce the previous calculations for this case and see that $\Delta V$ is always negative and no phase transition is possible.

\section{ISOSPECTRALITY}

All AdS/QCD outputs being at least indirectly connected to the spectra of the resonances, it is worth mentioning a recent proposal to modify the holographic models preserving the spectrum form. In Ref. [20], one finds such a study featuring a family of dilatons related to the usual SW one. The family is achieved through the notion of the isospectral potentials.

We propose investigating the way the predictions of the deconfinement temperature vary for different members of a dilaton family. In particular, we will adopt the method of Ref. [20] to the massless 5D fields subject to the EOM (10) and derive an extension to the case of GSW.

It is straightforward under a certain change of variables $\Phi(q, z)=f(z)^{-1} z^{(3-2 J) / 2} \Psi(q, z)$ to rewrite Eq. (10) for the KK $z$ profiles $\psi_{n}(z)$ in a Schrödinger form,

$$
-\psi_{n}^{\prime \prime}(z)+\hat{\mathcal{V}}(z) \psi_{n}(z)=M^{2}(n) \psi_{n}(z)
$$

Here $\hat{\mathcal{V}}(z)$ is the Schrödinger potential for a model with unspecified dilaton function $f^{2}(z)$. We further add a subscript $J$ because the potential is generally dependent on the spin parameter $J$ as follows,

$$
\hat{\mathcal{V}}_{J}(z)=\frac{(3-2 J)(5-2 J)}{4 z^{2}}+\frac{f^{\prime \prime}(z)}{f(z)}-\frac{3-2 J}{z} \frac{f^{\prime}(z)}{f(z)} .
$$


For the dilaton profile of the GSW model, it is given by

$$
\mathcal{V}_{J}(z)=\frac{(3-2 J)(5-2 J)}{4 z^{2}}+\kappa^{4} z^{2}+2 \kappa^{2}(1-J+2 b) \text {. }
$$

A particular form of the Schrödinger potential defines the eigenvalues of Eq. (26) and hence the mass spectrum $M(n)$. In the case of $(\mathrm{G}) \mathrm{SW}$ models, it is a potential similar to the one that appears when considering the radial part of the wave function of a two-dimensional harmonic oscillator system. The eigenvalues being known, we simultaneously gain the spectrum of Eq. (20).

According to Ref. [20] and the references therein, the following isospectral transformation between $\mathcal{V}_{J}(z)$ and $\hat{\mathcal{V}}_{J}(z)$ exists:

$$
\hat{\mathcal{V}}_{J}(z)=\mathcal{V}_{J}(z)-2 \frac{d^{2}}{d z^{2}} \ln \left[I_{J}(z)+\lambda\right] .
$$

This technique allows us to generate a family of dilaton functions $f(z)$ appearing in $\hat{\mathcal{V}}_{J}(z)$, each member assigned to the value of the parameter $\lambda$ (we assume $0<\lambda<\infty$ ). The case of $\lambda=\infty$ corresponds to the original $\mathcal{V}_{J}(z)$. The function $I_{J}(z)$ is defined through the ground eigenstate of $\mathcal{V}_{J}, \psi_{0}$ and is given by

$$
I_{J}(z) \equiv \int_{0}^{z} \psi_{0}^{2}\left(z^{\prime}\right) d z^{\prime}=1-\frac{\Gamma\left(|2-J|+1, \kappa^{2} z^{2}\right)}{\Gamma(|2-J|+1)} .
$$

Different $\lambda$ provide slightly different forms of the potential, but the eigenvalues of Eq. (26) and, hence, the spectrum remain the same. The given trick is well known in supersymmetric quantum mechanics [29] and represents a feature of one-dimensional potentials: the discrete spectrum of normalizable modes does not fix the potential, and there is an infinite family of such isospectral potentials that are related by the transformations of the kind (29). In this construction, the parameter $\lambda$ has no direct physical meaning and just reflects this specific spectral "symmetry" in the "space" of one-dimensional potentials.

We restrict the model to deviations only in the exponential factor, i.e., the isospectral profiles of a type $f^{2}(z)=$ $\exp (-\chi(z)) U^{2}\left(b, J-1 ; \kappa^{2} z^{2}\right)$, with the asymptotes fixed $\chi(z \rightarrow 0)=\chi(z \rightarrow \infty)=\kappa^{2} z^{2}$. Introducing an argument $t=\kappa^{2} z^{2}$, we may define the family of $\lambda$-dependent equations as follows:

$$
\begin{aligned}
\frac{t f^{\prime \prime}(t)+(J-1) f^{\prime}(t)}{f(t)}-b= & \frac{t+2(1-J)}{4}-\left(\frac{d}{d t}+2 t \frac{d^{2}}{d t^{2}}\right) \\
& \times \ln \left(I_{J}(t)+\lambda\right) .
\end{aligned}
$$

For a given $b, J$, and $\lambda$, an interpolating function for $\chi(\lambda, z)$ may be numerically found and substituted afterward inside $f^{2}(z)$ in the $\Delta V$ equation:

$$
\begin{aligned}
\Delta V_{\mathrm{GSW}}= & \frac{\pi R^{3}}{k_{g} z_{h}}\left[\frac{U^{2}(b, J-1,0)}{2}\right. \\
& -f^{2}\left(\kappa^{2} z_{h}^{2}\right)-\left.\kappa^{2} z_{h}^{2} \frac{d f^{2}(t)}{d t}\right|_{t=\kappa^{2} z_{h}^{2}} \\
& \left.-\left(\kappa z_{h}\right)^{4} \int_{\kappa^{2} z_{h}^{2}}^{\infty} d t t^{-1} \frac{d^{2} f^{2}(t)}{d t^{2}}\right] .
\end{aligned}
$$

As a family parameter $\lambda$ defines different $\chi(\lambda, z)$, the solutions of this equation and hence the critical temperature may vary. Are these deviations of a large scale, or does isospectrality preserve isothermality in general? If not, could we choose a correct family member with a "physical" value of the formal parameter $\lambda$ ? May be there are some specific fits to $(\mathrm{G}) \mathrm{SW}$ that provide more stable results than others. We suggest exploring these options in Sec. VI.

\section{LATTICE AND EXPERIMENT}

\section{A. Deconfinement temperature}

The deconfinement transition in the QCD matter is a complicated and largely not-understood process. Basically, we presume that with the temperature growth the description in terms of hadronic states becomes worse and worse until finally one should turn to consider the hadron matter as the quark-gluon plasma. One would like to define some critical parameter $T_{c}$ at which the change happens. Further, one may turn to the experimental data on heavy ion collisions, introduce the (model-dependent) way to extract the particular information, and achieve the constant value of $T_{c} \simeq 160 \mathrm{MeV}$ (e.g., see Ref. [30]).

Alternatively, a lot of investigations on the lattice have been performed to study the problem. They leave no doubt that the deconfinement happens smoothly with temperature and represents a crossover rather than a phase transition [24,31]. Hence, the notion of the critical parameter is substituted with the pseudocritical temperature, the precise determination of which is subject to the method and may vary in an almost 20\% range. The results of lattice simulations with physical quarks [24] provide the values $T_{c} \sim 150-170 \mathrm{MeV}$.

On the other hand, pure Yang-Mills theories (with no dynamical quarks at all) exhibit a somewhat similar phase structure. The confined phase corresponds to the bound glueball states, while the deconfined phase corresponds to the gluon plasma. Here the deconfinement happens as a true phase transition, and it is of a first order for $S U(3)$. Turning to the lattice studies of $S U(3)$ theory $[32,33]$, we encounter $T_{c} \sim 260-270 \mathrm{MeV}$. Lattice QCD simulations with nondynamical quarks in the limit $N_{c} \rightarrow \infty$ describe a similar physical system, and the prediction there is $T_{c} / \sqrt{\sigma}=$ $0.5949+0.458 / N_{c}^{2}$ [34]. The temperature, as well as other lattice outputs, is generically measured in terms of the dimensional quantity- the string tension $\sigma$. With the standard choice $\sqrt{\sigma}=420 \mathrm{MeV}$, we get $T_{c} \sim 250 \mathrm{MeV}$. 
The temperature estimations of the last paragraph should be the most relatable to the holographic predictions as it is defined in the leading $N_{c}$ order and as we associate the deconfinement holographic transition with the first order Hawking-Page phase transition. Nevertheless, it is a common practice to compare AdS/QCD predictions with the "real QCD" results of 150-170 MeV. The pro arguments here are that the strict $N_{c}=\infty$ limit is almost always softened to achieve real phenomenology, there is a relatively good description of hadron resonances in AdS/QCD, and the critical parameter turns to be a satisfactory approximation of the pseudocritical lattice one (based on particular numerical fits, some controversy about which is discussed in Sec. VI).

\section{B. Assumed $0^{++}$glueball states}

\section{Lattice calculations}

The lattice studies of glueball states and investigation of their masses were mostly performed in the quenched approximation, i.e., in the pure gluon theory. Further, we quote several works simulating $S U(3)$ on the lattice:

(i) Morningstar and Peardon [35] reported in 1999 on $0^{++}$[with a mass of $1730(50)(80) \mathrm{MeV}$ ] and $0^{++*}$ states that approximately follow the radial Regge trajectory $m^{2}=4 \cdot(1017 \mathrm{MeV})^{2} \cdot(n+0.72)$.

(ii) Meyer in Ref. [36] finds a ground state at 1475(30) (65) $\mathrm{MeV}$ and three radial excitations that belong nicely to a linear trajectory $m^{2}=4 \cdot(1094 \mathrm{MeV})^{2}$. $(n+0.46)$; see Fig. 2.

(iii) Chen et al. in Ref. [37] discuss the $0^{++}$state that has the mass of 1710(50)(80) MeV.

It is noted in Ref. [38] that the possible source of a systematic difference in the results of Meyer with respect to the ones of Morningstar and Peardon and Chen et al. for the ground state is the usage of the string tension $\sigma$ vs the hadronic scale parameter $r_{0}$ to determine the lattice spacing. However, one can notice from Fig. 2 that the full trajectory of Ref. [36] is in a general accordance with an interpolation of Ref. [35].

The large- $N_{c}$ limit is sometimes considered as well, as in practice the results for not so high a group degree, say $S U(8)$, do not alter much from the infinite $N_{c}$ extrapolation. We find the results of Ref. [39] the most trustworthy and quote them in terms of $\sigma: \frac{m^{2}}{\sigma}=4 \cdot 2.55 \cdot(n+0.42)$ (they are a revised version of the widely quoted Ref. [40]). In Ref. [36], the results for $S U(8)$ are claimed to be a valid approximation of the $N_{c} \rightarrow \infty$ limit and are given by $\frac{m^{2}}{\sigma}=4 \cdot 1.67 \cdot(n+0.99)$.

The unquenched approximation provides another interesting viewpoint. Though earlier it was supposed that glueball masses remain almost the same or get a $20-40 \%$ suppression with respect to the quenched results, the authors of the most recent work on this subject [38] report on the ground state of $1795(60) \mathrm{MeV}$, which is greater than any

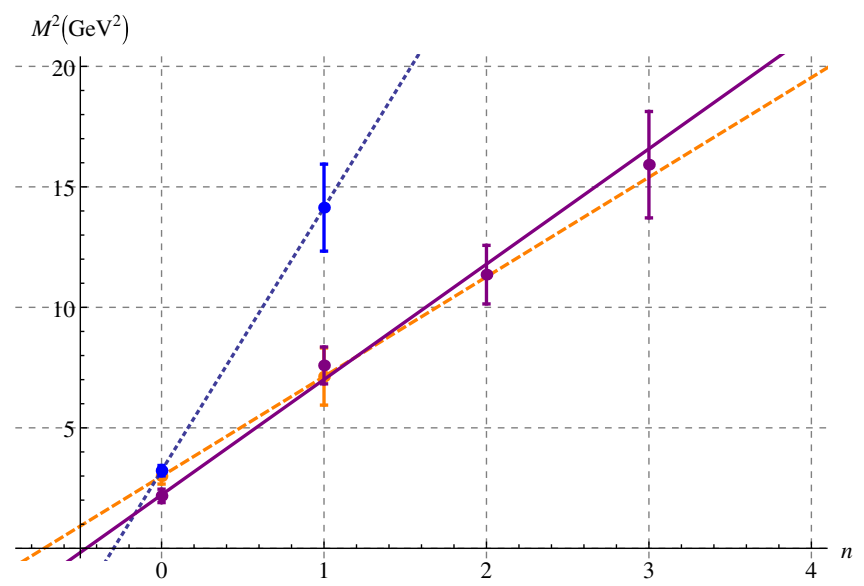

FIG. 2. The radial Regge trajectories of glueball states: dashed is Morningstar and Peardon [35], solid is Meyer [36], and dotted is unquenched [38].

aforementioned value. Together with the first excitation, the unquenched method of Ref. [38] provides a trajectory $m^{2}=4 \cdot(1652 \mathrm{MeV})^{2} \cdot(n+0.30)$. Interestingly, the slope is much steeper than in the quenched approximation due to the $0^{++*}$ state of Ref. [38] being in the range of $0^{++* * *}$ of Ref. [36], as is clear from Fig. 2.

\section{Candidate among $f_{0}(1370), f_{0}(1500)$, and $f_{0}(1710)$}

Having established from the lattice a region of masses where the scalar glueball may be encountered, $1.5-1.7 \mathrm{GeV}$, one looks there for the nonstrange mesons with the same quantum numbers that do not fit into $q \bar{q}$ nonets. The most often regarded hypothesis emerges then: $f_{0}(1370)$, $f_{0}(1500)$, and $f_{0}(1710)$ states are a mixture of $\bar{u} u+\bar{d} d$, $\bar{s} s$, and glueball modes.

However, a common viewpoint on the degree of the mixing, or on what state could be mostly glueballic, does not exist. For a variety of possibilities, in broader mass ranges as well, see, for instance, Ref. [4]. An attempt to separate in the set of known $f_{0}$ 's the radial Regge trajectory of mesonic states from the glueballs from the point of view of rotated closed strings in the holographic background was made by the authors of Ref. [41]. Though various interesting fits to different glueball candidates are presented there, there is no final conclusion but that "an extension of experimental data on the spectrum of flavorless hadrons is needed" [41]. In this paper, we do not try to cover all the options and will focus on the two main ones.

The first model, rather strongly advocated by the Particle Data Group [3], is assuming that $f_{0}(1500)$ has the largest glueball component. $f_{0}(1370)$ consists mostly of the up and down quarks, and $f_{0}(1710)$ consists of the strange ones. For a typical example, we further take the results of Ref. [42], in which two fits to existing data are provided: fit I gives $m_{g l}=1464 \pm 47 \mathrm{MeV}$, and fit II gives $m_{g l}=$ $1519 \pm 41 \mathrm{MeV}$. 


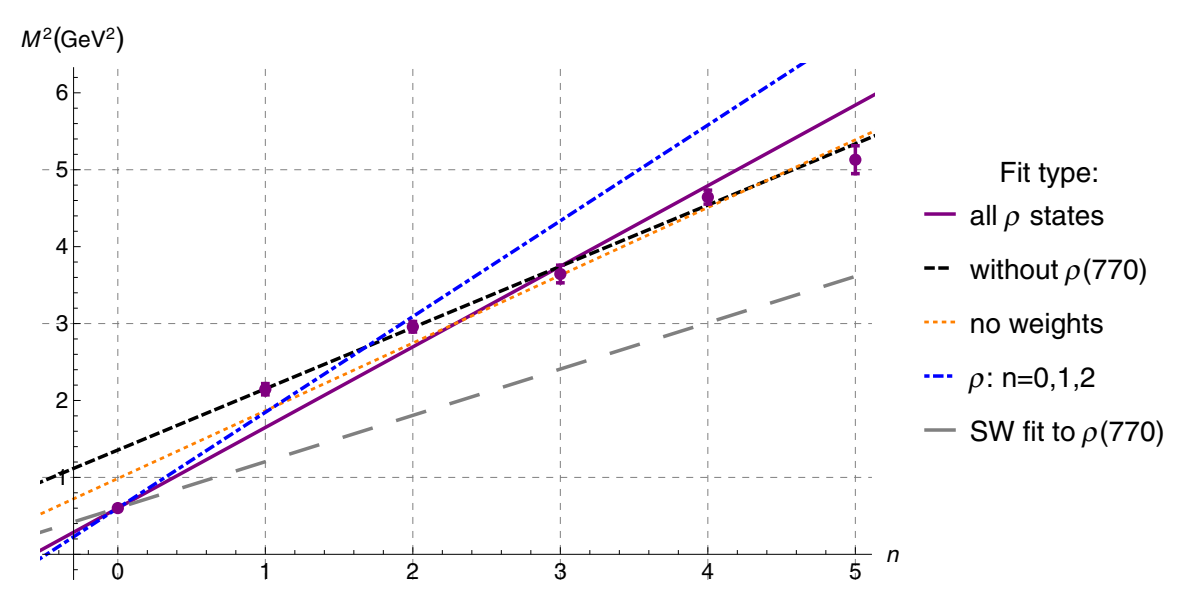

FIG. 3. The possibilities for the radial Regge trajectories of the $\rho$ meson.

The second model proposes exchanging the roles of $f_{0}(1500)$ and $f_{0}(1710)$ and, suggesting that the latter has a predominant glueball component, gives $m_{g l} \sim 1665 \mathrm{MeV}$ [43]. A revised version of this model [44] provides $m_{g l}=$ $1674 \pm 14 \mathrm{MeV}$.

Shortly, the first model is mostly supported by the fact that $f_{0}(1500)$ are not encountered in $\gamma \gamma$ reactions. At the same time, the authors of Ref. [44] argue that this fact does not necessarily imply a large glueball component there and advocate the $f_{0}(1710)$ option from the point of $J / \psi$ decays. It is also concluded that $f_{0}(1710)$ is a ground glueball state in some theoretical works $[9,45]$.

\section{Vector mesons}

The $\rho$ and $\omega$ mesons, despite being seemingly welldefined particles, pose certain trouble for one aspiring to explore the spectrum of their radial excitations in much detail: the ground state lying notably below the linear trajectory, broad resonances, etc. See Ref. [16] for some discussion on these issues.

The ground state poses the main problem in the present study as, on one hand, it has the most established mass value, being most useful for the numerical estimations, but on the other hand, it deviates significantly from the linear trajectory of the higher radial excitations; see Fig. 3. The latter fact questions and obscures the usage of these trajectories in the SW-like models. It should be mentioned that there are many specific modifications of the SW model that lead to certain nonlinearities of radial trajectories: dynamical [46] and nondynamical $[47,48]$ modifications of the dilaton and AdS metric as well as SW models containing a cutoff of the holographic coordinate [49]. Nevertheless, we try to make several realistic hypotheses with linear trajectories in the following section.

\section{HOLOGRAPHIC PREDICTIONS FOR $\boldsymbol{T}_{c}$}

A general disclaimer for this section is that we consider all numerical results at the level of an estimation. The appearing error bars are only due to the uncertainty of the experimental or lattice determination of the input parameters. The holographic apparatus can only claim numerical validity on the semiquantitative level, and we cannot wholly estimate the theoretical error. Though for the well-known masses of the vector mesons the experimental error is definitely small and not comparable to the theoretical one, the lattice results are not that precise, and we find it useful to provide the minimal possible error that could be present.

\section{A. Deconfinement temperature from the vector channel}

Eventually, we are going to discuss the numerical holographic predictions on $T_{c}$ and start with the vector case, $J=1$.

In Table I, we cover the results, some of which were previously given in Refs. $[15,16]$. The first two lines represent the classical AdS/QCD fit with the lightest state (usually a $\rho$ meson), while the third represents the fit to the tower of the established resonances (the ground state and the first and second radial excitations). It is evident from Fig. 3 that the SW fit to the ground state does not follow the real trajectory of the $\rho$ excitations; neither does the HW spectrum, which goes as $M(0)\{1 ; 2.3 ; 3.6 ; \ldots\}$, featuring

TABLE I. Standard vector meson predictions for the deconfinement temperature in $\mathrm{HW}$ and (G)SW.

\begin{tabular}{|c|c|c|c|}
\hline \multirow[b]{2}{*}{ Fit and method } & \multicolumn{3}{|c|}{$T_{c}(\mathrm{MeV})$} \\
\hline & $\rho$ meson & $\omega$ meson & $\begin{array}{l}\text { Universal } \\
\text { trajectory }\end{array}$ \\
\hline $\begin{array}{c}\text { Lightest state } \\
\text { and HW }\end{array}$ & $122.03 \pm 0.04$ & $123.19 \pm 0.02$ & $168.1 \pm 0.7$ \\
\hline $\begin{array}{l}\text { Lightest state } \\
\text { and SW }\end{array}$ & $190.61 \pm 0.06$ & $192.43 \pm 0.03$ & $262.5 \pm 1.2$ \\
\hline $\begin{array}{c}n=0,1,2 \\
\text { and GSW }\end{array}$ & $118.3 \pm 2.7$ & $121.4 \pm 4.0$ & $\cdots$ \\
\hline
\end{tabular}


much faster growth than the actual $\rho$ spectrum $M(\rho)$ $\{1 ; 1.9 ; 2.0 ; \ldots\}$. One would expect the inclusion of higher excitations to help with the spectrum description, but it does not happen due to the high weight of the ground state in the fit. For instance, the $n=0,1,2$ trajectory deviates significantly from the further states and provides the temperature estimation of $118 \mathrm{MeV}$ that does not seem satisfying. The same can be attributed to the $n=0,1,2$, 3, 4 fit resulting in $T_{c} \simeq 125 \mathrm{MeV}$. Thus, all the $T_{c}$ predictions of Table I from $\rho$ and $\omega$ fits rely on the nonstandard position of the lightest state and do not come close to any lattice estimate.

One way around this to include equal weights in the fit as was performed in Ref. [16] in hopes of getting an average fit that can bring better results for the temperature estimations. Indeed, this strategy resulted in $T_{c} \simeq 150 \mathrm{MeV}$ for $n=0,1,2$ trajectories.

Another option is presented in the third column of Table I. As argued in Ref. [16], we find the fit with "universal" slope to be the most successful. There are some phenomenological reasons for the universality of the slope for various light nonstrange mesons as noticed in Refs. [21,50] and further advocated in many studies [51]. As well the universality of this kind is in concordance with the hypothesis (inspired by the hadron string models) that the slope is mainly determined by the gluodynamics. From the holographic $(\mathrm{G}) \mathrm{SW}$ model point of view, the universality of the slope means the universality of the dilaton profile for the particles of any spin. Moreover, the use of the universal slope $(\kappa=534 \mathrm{MeV}$ [21]) provides a unique $T_{c} \simeq 260 \mathrm{MeV}$. This result being in range of lattice predictions with nondynamical quarks allows us to claim that the fit defined from a gluodynamic insight provides the deconfinement temperature expected from gluodynamics. It was highlighted in the Introduction and can be seen below that this fit corresponds to selecting the $f_{0}(1500)$ meson as the lightest scalar glueball candidate.

Now, let us consider the families of isospectral dilatons and their impact on the value of $T_{c}$, which is provided in Table II. It is clear that for vector mesons isospectrality does not come together with isothermality. There are two ways to treat this problem. First is to say that, taking into account the scale of changes in $T_{c}$ with different $\lambda$, none of the results on the deconfinement temperature in this model is trustworthy. Another is to consider that if holography exists in nature there could be various reasons for $\lambda$ to take a particular "true" value, not essentially the original $\lambda=\infty$. What other physical processes affect the particular choice of $\lambda$ corresponding to the true $T_{c}$ may be a subject of future investigations.

We have chosen several noteworthy examples in Table II. For the universal trajectory, we see a transition of the $T_{c}$ from the region predicted on lattice with nondynamical quarks (about $260 \mathrm{MeV}$ ) to the one of the unquenched approximation (150-170 MeV). For the standard SW model, we can get a viable prediction at some finite $\lambda$. For the GSW, we take a specific fit trying to specify the role of $\rho(770)$-we just exclude the ground state (see Fig. 3). Although at $\lambda=\infty$ this trajectory provides a very large and unnatural $T_{c}$, we can go down to the expected values at finite $\lambda$. At least this consideration allows us to avoid the complete exclusion of the higher $\rho$ trajectory hypothesis following the criterion of viable $T_{c}$ predictions.

\section{B. Deconfinement temperature from the glueball channel}

To begin with scalar glueball estimations of $T_{c}$, we propose combining lattice results in the $N_{c} \rightarrow \infty$ limit for the relation of $T_{c}$ to $m_{g l}$, where $m_{g l}$ is the mass of the $0^{++}$ state. Both quantities are measured on the lattice in terms of $\sqrt{\sigma}$, so such a fraction provides a $\sqrt{\sigma}$-independent result. From Refs. [34,39], we get

$$
\left.\frac{T_{c}}{m_{\mathrm{gl}}}\right|_{\mathrm{lat}}=0.1799
$$

while the HW and SW predictions are

$$
\left.\frac{T_{c}}{m_{\mathrm{gl}}}\right|_{\mathrm{HW}}=0.0988,\left.\quad \frac{T_{c}}{m_{\mathrm{gl}}}\right|_{\mathrm{SW}}=0.1739 .
$$

The SW result is rather close, much better than the one achieved in the models of improved holographic QCD: $\left.\frac{T_{c}}{m_{\mathrm{gl}}}\right|_{\mathrm{IhQCD}}=0.167$ [52]. If both states reported in Ref. [39] are considered and supposed to lie on the GSW trajectory,

TABLE II. The isospectral parameter $\lambda$ affecting the deconfinement temperature estimations from vector meson fits in $(\mathrm{G}) \mathrm{SW}$ models.

\begin{tabular}{lcccc}
\hline \hline & \multicolumn{2}{c}{$T_{\mathrm{SW}}(\mathrm{MeV})$} & & $T_{\mathrm{GSW}}(\mathrm{MeV})$ \\
\cline { 2 - 3 }$\lambda$ & $\begin{array}{c}\text { Universal trajectory } \\
m^{2}=4 \cdot(534 \mathrm{MeV})^{2} \cdot(n+1)\end{array}$ & $\begin{array}{c}\text { Lightest } \rho \text { meson } \\
m^{2}=4 \cdot(388 \mathrm{MeV})^{2} \cdot(n+1)\end{array}$ & $\begin{array}{c}\rho: n=1,2,3,4,5[3] \\
m^{2}=4 \cdot(446 \mathrm{MeV})^{2} \cdot(n+1.7)\end{array}$ \\
\hline 100 & $261.1 \pm 1.2$ & $189.66 \pm 0.06$ & $422.5 \pm 34.2$ \\
20 & $256.1 \pm 1.1$ & $185.97 \pm 0.06$ & $419.0 \pm 34.5$ \\
1 & $194.9 \pm 0.9$ & $141.54 \pm 0.05$ & $339.2 \pm 39.2$ \\
0.1 & $159.1 \pm 0.7$ & $115.53 \pm 0.04$ & $189.2 \pm 11.4$ \\
0.01 & $154.3 \pm 0.7$ & $112.03 \pm 0.04$ & & $172.4 \pm 8.0$ \\
\hline \hline
\end{tabular}


TABLE III. Dependence of the deconfinement temperature estimations from vector meson fits in the $(\mathrm{G}) \mathrm{SW}$ model on the isospectral parameter $\lambda$

\begin{tabular}{|c|c|c|c|c|c|}
\hline \multirow[b]{2}{*}{ Fit } & \multirow[b]{2}{*}{$m_{\mathrm{gl}}(\mathrm{MeV})$} & \multirow[b]{2}{*}{$T_{\mathrm{HW}}(\mathrm{MeV})$} & \multicolumn{3}{|c|}{$T_{\mathrm{SW}}(\mathrm{MeV})$} \\
\hline & & & $\lambda=\infty$ & $\lambda=1$ & $\lambda=0.1$ \\
\hline Morningstar and Peardon [35] & $1730(100)$ & 171(10) & 301(17) & $253(15)$ & $173(10)$ \\
\hline Meyer [36] & $1475(75)$ & $146(7)$ & $256(13)$ & $215(11)$ & 147(8) \\
\hline Chen et al. [37] & $1710(95)$ & $169(9)$ & 297(17) & $250(14)$ & $171(10)$ \\
\hline Large $N_{c}[39]$ & $1455(70)$ & $144(7)$ & $253(12)$ & $212(10)$ & $145(7)$ \\
\hline Unquenched [38] & $1795(60)$ & 177(6) & $312(10)$ & $262(9)$ & $179(6)$ \\
\hline \multirow[t]{2}{*}{$f_{0}(1500)$ meson [42] } & $1464(47)$ & $145(5)$ & $255(8)$ & $214(7)$ & $146(5)$ \\
\hline & $1519(41)$ & $150(4)$ & $264(7)$ & $222(6)$ & $152(4)$ \\
\hline$f_{0}(1710)$ meson $[44]$ & $1674(14)$ & $165(1)$ & 291(2) & $244(2)$ & $167(1)$ \\
\hline
\end{tabular}

TABLE IV. Predictions of $T_{c}$ from different fits for glueball towers in the GSW model with isospectrality.

\begin{tabular}{|c|c|c|c|c|c|c|}
\hline \multirow[b]{2}{*}{ Fit } & \multirow[b]{2}{*}{$\sqrt{\sigma}$ or $r_{0}^{-1}(\mathrm{MeV})$} & \multicolumn{2}{|c|}{$m^{2}=4 \kappa^{2}(n+2+b)$} & \multicolumn{3}{|c|}{$T_{\mathrm{GSW}}(\mathrm{MeV})$} \\
\hline & & $\kappa(\mathrm{MeV})$ & $\mathrm{b}$ & $\lambda=\infty$ & $\lambda=1$ & $\lambda=0.1$ \\
\hline Morningstar and Peardon [35] & 410 & $1017(151)$ & $-1.28(0.23)$ & $153.6(39.2)$ & $151.4(36.1)$ & $149.9(34.1)$ \\
\hline Meyer [36] & 440 & 1094(49) & $-1.54(0.07)$ & $132.5(9.7)$ & $132.3(9.5)$ & $132.1(9.4)$ \\
\hline Unquenched [38] & 420 & $1652(138)$ & $-1.71(0.05)$ & $174.6(16.4)$ & $174.6(16.4)$ & $174.5(16.4)$ \\
\hline Large $N_{c}[39]$ & 440 & $1120(88)$ & $-1.58(0.08)$ & $131.3(13.5)$ & $131.1(13.4)$ & $131.0(13.3)$ \\
\hline Large $N_{c}$ [36] & 440 & $735(121)$ & $-1.00(0.35)$ & $142.8(55.3)$ & $134.8(43.7)$ & $129.7(36.6)$ \\
\hline
\end{tabular}

we get $\frac{T_{c}}{\sqrt{\sigma}}=0.2988$ to be compared with 0.5949 of Ref. [34]. This does not seem satisfying, but remarkably, isospectral methods change this result only in the last digit.

Utilizing the formulas of Eq. (34) and taking the $0^{++}$ glueball mass in the physical scale, we produce the variety of results of Table III. The isospectrality is considered for the SW model and features behavior similar to what we have seen in the vector case. The $\mathrm{HW}$ results converge to a region of 150-170 MeV, close to the lattice with dynamical quarks. The SW predictions are a bit larger than the quenched lattice ones, though it is possible to get lower within the isospectral family. Both fits of the hypothesis of $f_{0}(1500)$ being a mostly glueball state predict $\sim 260 \mathrm{MeV}$ in the SW and generally are close to the fit of the universal vector trajectory. The large- $N_{c}$ fit provides a similar number $\sim 250 \mathrm{MeV}$ in the $\mathrm{SW}$, showing nice concordance with large- $N_{c}$ predictions from the lattice.

Next, in Table IV, we take the full spectra of radial excitations and define $T_{c}$ through GSW formulas to achieve astonishing isothermality. The error bars here are much larger as the higher glueball excitations are not that well measured and usually only the masses of $0^{++}$and $0^{++*}$ states are calculated. First, that does not allow us to be sure of the validity of linear Regge assumption for the radial excitations of the scalar glueballs. Second, the slope error may get rather significant. However, the only work reporting on more than two states, Ref. [36], gives a more well-defined trajectory (and rather linear; see Fig. 2).
The $T_{c}$ prediction there is rather low, but that is due to the fact that the ground state is calculated to have a mass $\sim 1.5 \mathrm{GeV}$, which may be considered systematically lower than other lattice predictions for the masses. Altogether, we find all $T_{c}$ of Table IV to be rather close to the results of unquenched lattice predictions for the deconfinement temperature, and it is especially significant to have this agreement for the unquenched fit of Ref. [38].

\section{CONCLUDING DISCUSSIONS}

A general motivation of this paper is to raise awareness that, while making AdS/QCD calculations, one should be concerned from where and for what reasons one takes the input parameters.

We can easily assume an occurrence of different dilaton prefactors in the gravity and matter parts of the 5D action. Moreover, for any scalar, vector, and tensor operators in a given strongly interacting theory, we may construct a $5 \mathrm{D}$ Lagrangian with a suitable 5D field following the AdS/CFT dictionary. If we want to consider a holographic description involving all this physics (at the level the bottom-up holography can manage), we should construct a general Lagrangian of the type

$$
\begin{aligned}
S_{5 D}= & \int d^{5} x \sqrt{-g}\left(f_{\text {gr }}^{2}(z) \mathcal{L}_{\text {gravity }}+\sum_{j} f_{s c j}^{2}(z) \mathcal{L}_{\text {scalar } j}\right. \\
& \left.+\sum_{j} f_{v j}^{2}(z) \mathcal{L}_{\text {vector } j}+\cdots\right)
\end{aligned}
$$


Here, $f^{2}(z)$ schematically means HW, (G)SW, or any other $z$-dependent prefactor induced by extra-dimensional dynamics together with the restrictions on the $z$ interval. We could generally assume that all $f_{j}^{2}(z)$ are different. This option is not theoretically well motivated as the dilaton field is a certain entity of the string theory, and, for instance, in the models of dynamically generated SW, it comes as a unique solution of Einstein equations emerging from a particular graviton-dilaton 5D action (see Refs. [53,54] for the interesting results concerning glueballs in the models of this type). But phenomenologically, this only seems natural, and, e.g., the case of $\kappa_{s c}=\kappa_{v}$ in a standard $\mathrm{SW}$ is a feature that may be or may be not relevant in the physical model we want to study.

Then, why should the gravity dilaton be coincident with some other? Generally, from the bottom-up point of view only, it should not and may be considered as another free parameter. Nevertheless, it is our main objective to determine the region to which the value of $T_{c}$ belongs, and the model predictability cannot be given up so easily.

We find no other option than to try to consider several possibilities and determine the "correct" one (if it exists) not only for the conceptual reasons but also for the phenomenological ones. An expense of free parameters not being a feature we require, we utilize the least finetuned holographic frameworks: HW, SW, and GSW. For the matter content, we make the traditional choice of vector mesons and the one related to the similarity of the $N_{c}$ scaling of 4D gluodynamics and 5D gravitational action, i.e., $0^{++}$glueball and its radial excitations. For an optional check in SW-like models, we propose aspiring to constant temperature predictions in an isospectral family.

For a minimal option, we argue once more on the relevance of the idea of the universal slope value for the radial trajectories of light nonstrange mesons. That means a fixation of the dilaton parameter in $f_{g r}^{2}(z)=e^{-\kappa^{2} z^{2}}$ to $\kappa \simeq 530 \mathrm{MeV}$, which coincides with the case of $f_{0}(1500)$ being predominantly a $0^{++}$glueball. This results in $T_{c} \simeq$ $260 \mathrm{MeV}$ corresponding exceedingly well to the lattice results for pure $S U(3)$ and the large- $N_{c}$ limit. Both heuristics and numbers are in favor of this hypothesis. Moreover, this is the least model-dependent result in our considerations. The lack of isothermality in the isospectral family of this dilaton seems to be the only downside.

The simplest models, HW and SW, feature a rough fitting to the radial trajectories as a whole (especially in HW), and it is convenient to restrict them to reproduce the masses of the ground states. For scalar glueballs, it is even more essential, as the ground states are much better identified on lattice than their excitations. We find that for $0^{++}$glueball candidates (from lattice or from the identification with some $f_{0}$ ), the masses of which are limited within the range of $1.5-1.7 \mathrm{GeV}$, the deconfinement temperature lies in the range of 260 $290 \mathrm{MeV}$ in the SW and $150-170 \mathrm{MeV}$ in the HW. For the $\rho$ or $\omega$ mesons, we get $\sim 190 \mathrm{MeV}$ in the SW and $\sim 120 \mathrm{MeV}$ in the HW. Clearly, the results of the HW and SW differ, though for the glueballs, they appear to coincide with lattice expectations in different regimes. That is difficult to interpret, though we can notice that going through the isospectral family we can connect these separated regions (perhaps, in a way, mimicking quark masses becoming physical on lattice).

The generalized SW models provide a more accurate description of the excited spectrum. We studied the predictions from the various spectra of vector mesons in Ref. [16]. There, we have seen that different spectra may result in various predictions for $T_{c}$, not providing a clear way to select the best fit. Here, we find out that additionally these temperature estimates vary a lot as we go through the isospectral family. In this work, we performed a similar analysis for the scalar glueballs and realized that, first, the isothermality is automatically achieved, and, second, that the predicted values are close to the unquenched lattice estimations of $T_{c}$. The accordance of the unquenched glueball spectrum fit giving $T_{c} \simeq 175 \pm 15 \mathrm{MeV}$ appears particularly successful in our view.

In conclusion, we suppose that our analysis contains several new observations concerning the calculation of the deconfinement temperature in AdS/QCD. At the same time, it is not meant to be a criticism of Ref. [15] and its followers (as we exploited a similar treatment in Ref. [16]). Rather, we consider this as a possibility to clarify the methodology and broaden the horizons of (semi)quantitative bottom-up holographic estimations.

We do not provide any new theoretical insight on why the first-order Hawking-Page phase transition should reproduce the crossover nature of the QCD deconfinement process or on the string theory configurations leading to the GSW models. But we attempted, within some of these models, to make an honest estimation of the quantity of much discussion in the modern physics in these models that may not be perfect but do retain some predictive power.

For the development of this work, we see several directions. First, we may try to be not limited by only scalar glueballs and consider pomeron/odderon Regge trajectories. Second, we may study various ways to include other axes (vector, axial and isospin quark chemical potentials, magnetic field) to the holographically generated phase diagram. 
[1] H. Fritzsch and M. Gell-Mann, Current algebra: Quarks and what else?, arXiv:hep-ph/0208010.

[2] H. Fritzsch and P. Minkowski, $\Psi$ Resonances, gluons and the Zweig rule, Nuovo Cimento A 30, 393 (1975).

[3] M. Tanabashi et al. (Particle Data Group), Review of particle physics, Phys. Rev. D 98, 030001 (2018).

[4] W. Ochs, The status of glueballs, J. Phys. G 40, 043001 (2013).

[5] D. Parganlija, Mesons, PANDA and the scalar glueball, J. Phys. Conf. Ser. 503, 012010 (2014).

[6] D. Parganlija, Glueballs and vector mesons at NICA, Eur. Phys. J. A 52, 229 (2016).

[7] R. C. Brower, S. D. Mathur, and C.-I. Tan, Glueball spectrum for QCD from AdS supergravity duality, Nucl. Phys. B587, 249 (2000).

[8] K. Hashimoto, C.-I. Tan, and S. Terashima, Glueball decay in holographic QCD, Phys. Rev. D 77, 086001 (2008).

[9] F. Brünner, D. Parganlija, and A. Rebhan, Glueball decay rates in the Witten-Sakai-Sugimoto model, Phys. Rev. D 91, 106002 (2015); Erratum 93, 109903 (2016).

[10] H. Boschi-Filho and N. R.F. Braga, QCD/string holographic mapping and glueball mass spectrum, Eur. Phys. J. C 32, 529 (2004).

[11] P. Colangelo, F. D. Fazio, F. Jugeau, and S. Nicotri, On the light glueball spectrum in a holographic description of QCD, Phys. Lett. B 652, 73 (2007).

[12] H. Forkel, Holographic glueball structure, Phys. Rev. D 78, 025001 (2008).

[13] P. Colangelo, F. De Fazio, F. Jugeau, and S. Nicotri, Investigating AdS/QCD duality through scalar glueball correlators, Int. J. Mod. Phys. A 24, 4177 (2009).

[14] H. Boschi-Filho, N. R. F. Braga, F. Jugeau, and M. A. C. Torres, Anomalous dimensions and scalar glueball spectroscopy in AdS/QCD, Eur. Phys. J. C 73, 2540 (2013).

[15] C. P. Herzog, A Holographic Prediction of the Deconfinement Temperature, Phys. Rev. Lett. 98, 091601 (2007).

[16] S. S. Afonin and A. D. Katanaeva, Holographic Estimates of the Deconfinement Temperature, Eur. Phys. J. C 74, 3124 (2014).

[17] J. Erlich, E. Katz, D. T. Son, and M. A. Stephanov, QCD and a Holographic Model of Hadrons, Phys. Rev. Lett. 95, 261602 (2005).

[18] L. Da Rold and A. Pomarol, Chiral symmetry breaking from five dimensional spaces, Nucl. Phys. B721, 79 (2005).

[19] A. Karch, E. Katz, D. T. Son, and M. A. Stephanov, Linear confinement and AdS/QCD, Phys. Rev. D 74, 015005 (2006).

[20] A. Vega and P. Cabrera, Family of dilatons and metrics for AdS/QCD models, Phys. Rev. D 93, 114026 (2016).

[21] D. Bugg, Four sorts of meson, Phys. Rep. 397, 257 (2004).

[22] S. S. Afonin, Properties of new unflavored mesons below 2.4-GeV, Phys. Rev. C 76, 015202 (2007).

[23] S. S. Afonin, Light meson spectrum and classical symmetries of QCD, Eur. Phys. J. A 29, 327 (2006).

[24] S. Borsanyi, Z. Fodor, C. Hoelbling, S. D. Katz, S. Krieg, C. Ratti, and K. K. Szabo (Wuppertal-Budapest Collaboration), Is there still any T_c mystery in lattice QCD? Results with physical masses in the continuum limit III, J. High Energy Phys. 09 (2010) 073.
[25] S. J. Brodsky, G. F. de Teramond, H. G. Dosch, and J. Erlich, Light-front holographic QCD and emerging confinement, Phys. Rep. 584, 1 (2015).

[26] S. S. Afonin, Generalized soft wall model, Phys. Lett. B 719, 399 (2013).

[27] S. S. Afonin, Soft-wall modelling of meson spectra, Acta Phys. Pol. B Proc. Suppl. 9, 597 (2016).

[28] A. Karch, E. Katz, D. T. Son, and M. A. Stephanov, On the sign of the dilaton in the soft wall models, J. High Energy Phys. 11 (2011) 66.

[29] F. Cooper, A. Khare, and U. Sukhatme, Supersymmetry and quantum mechanics, Phys. Rep. 251, 267 (1995).

[30] A. Andronic et al., Hadron production in ultra-relativistic nuclear collisions: Quarkyonic matter and a triple point in the phase diagram of QCD, Nucl. Phys. A837, 65 (2010).

[31] Y. Aoki, G. Endrodi, Z. Fodor, S. D. Katz, and K. K. Szabo, The order of the quantum chromodynamics transition predicted by the standard model of particle physics, Nature (London) 443, 675 (2006).

[32] G. Boyd, J. Engels, F. Karsch, E. Laermann, C. Legeland, M. Lutgemeier, and B. Petersson, Thermodynamics of $S U(3)$ lattice gauge theory, Nucl. Phys. B469, 419 (1996).

[33] Y. Iwasaki, K. Kanaya, T. Kaneko, and T. Yoshie, Scaling of the critical temperature and quark potential with a renormalization group improved $S U(3)$ gauge action, Nucl. Phys. B, Proc. Suppl. 53, 429 (1997).

[34] B. Lucini, A. Rago, and E. Rinaldi, $S U\left(N_{c}\right)$ gauge theories at deconfinement, Phys. Lett. B 712, 279 (2012).

[35] C. J. Morningstar and M. J. Peardon, The glueball spectrum from an anisotropic lattice study, Phys. Rev. D 60, 034509 (1999).

[36] H. B. Meyer, Glueball Regge trajectories, Glueball regge trajectories, Ph.D. thesis, Oxford University, 2004.

[37] Y. Chen et al., Glueball spectrum and matrix elements on anisotropic lattices, Phys. Rev. D 73, 014516 (2006).

[38] E. Gregory, A. Irving, B. Lucini, C. McNeile, A. Rago, C. Richards, and E. Rinaldi, Towards the glueball spectrum from unquenched lattice QCD, J. High Energy Phys. 10 (2012) 170.

[39] B. Lucini, M. Teper, and U. Wenger, Glueballs and k-strings in $\mathrm{SU}(\mathrm{N})$ gauge theories: Calculations with improved operators, J. High Energy Phys. 06 (2004) 012.

[40] B. Lucini and M. Teper, $S U(N)$ gauge theories in fourdimensions: Exploring the approach to $\mathrm{N}=$ infinity, J. High Energy Phys. 06 (2001) 050.

[41] J. Sonnenschein and D. Weissman, Glueballs as rotating folded closed strings, J. High Energy Phys. 12 (2015) 1.

[42] F. E. Close and Q. Zhao, Production of $f_{0}(1710), f_{0}(1500)$, and $f_{0}(1370)$ in $J / \psi$ hadronic decays, Phys. Rev. D 71, 094022 (2005).

[43] H.-Y. Cheng, C.-K. Chua, and K.-F. Liu, Scalar glueball, scalar quarkonia, and their mixing, Phys. Rev. D 74, 094005 (2006).

[44] H.-Y. Cheng, C.-K. Chua, and K.-F. Liu, Revisiting scalar glueballs, Phys. Rev. D 92, 094006 (2015).

[45] S. Janowski, F. Giacosa, and D. H. Rischke, Is $f_{0}(1710)$ a glueball?, Phys. Rev. D 90, 114005 (2014).

[46] S. P. Bartz and J. I. Kapusta, Dynamical three-field AdS/ QCD model, Phys. Rev. D 90, 074034 (2014). 
[47] S. S. Afonin, AdS/QCD models describing a finite number of excited mesons with Regge spectrum, Phys. Lett. B 675, 54 (2009).

[48] L.-X. Cui, Z. Fang, and Y.-L. Wu, Infrared-improved softwall AdS/QCD model for mesons, Eur. Phys. J. C 76, 22 (2016).

[49] S. S. Afonin, Low-energy holographic models for QCD, Phys. Rev. C 83, 048202 (2011).

[50] A. V. Anisovich, V. V. Anisovich, and A. V. Sarantsev, Systematics of $q \bar{q}$ states in the $\left(n, M^{2}\right)$ and $\left(J, M^{2}\right)$ planes, Phys. Rev. D 62, 051502 (2000).

[51] E. Klempt and A. Zaitsev, Glueballs, hybrids, multiquarks: Experimental facts versus QCD inspired concepts, Phys. Rep. 454, 1 (2007); M. Shifman and A. Vainshtein, Highly excited mesons, linear Regge trajectories, and the pattern of the chiral symmetry realization, Phys. Rev. D 77, 034002 (2008); S. S. Afonin, Experimental indication on chiral symmetry restoration in meson spectrum, Phys. Lett. B 639, 258 (2006); Towards understanding spectral degeneracies in nonstrange hadrons. Part I. Mesons as hadron strings versus phenomenology, Mod. Phys. Lett. A 22, 1359 (2007); Parity doubling in particle physicsInt. J. Mod. Phys. A 22, 4537 (2007); Hydrogen like classification for light nonstrange mesons23, 4205 (2008); Implications of the crystal barrel data for meson-baryon symmetries, Mod. Phys. Lett. A 23, 3159 (2008); D. M. Li, B. Ma, Y. X. Li, Q. K. Yao, and H. Yu, Meson spectrum in Regge phenomenology, Eur. Phys. J. C 37, 323 (2004); P. Masjuan, E. R. Arriola, and W. Broniowski, Systematics of radial and angular-momentum Regge trajectories of light nonstrange $q \bar{q}$-states, Phys. Rev. D 85, 094006 (2012); S. S. Afonin and I. V. Pusenkov, Universal description of radially excited heavy and light vector mesons, Phys. Rev. D 90, 094020 (2014); S. S. Afonin and I. V. Pusenkov, Note on universal description of heavy and light mesons, Mod. Phys. Lett. A 29, 1450193 (2014); P. Masjuan, E. R. Arriola, and W. Broniowski, Hadron form factors and large- $N_{c}$ phenomenology, Eur. Phys. J. Web Conf. 73, 04021 (2014).

[52] U. Gursoy, E. Kiritsis, L. Mazzanti, G. Michalogiorgakis, and F. Nitti, Improved Holographic QCD, Lect. Notes Phys. 828, 79 (2011).

[53] D. Li and M. Huang, Dynamical holographic QCD model for glueball and light meson spectra, J. High Energy Phys. 11 (2013) 088.

[54] S. P. Bartz, A. Dhumuntarao, and J. I. Kapusta, Dynamical AdS/Yang-Mills model, Phys. Rev. D 98, 026019 (2018). 